\title{
SENSORIX AG
}

www.sensorix.ch

\section{Development, Optimization, and Control of Biotechnological Processes Through Continuous Online Analytics with Chemical Sensors and Biosensors}

\author{
Markus Rothmaier*
}

\begin{abstract}
The four-year-old ETH Zürich spinoff company SENSORIX develops, produces and sells analytical instrumentation for process development, optimization and control in biotechnology. SENSORIX successfully launched its first products for fermentation and cell culture applications last year. Based on a flexible and modular platform for biosensors and chemical sensors they can be tailored to match the changing needs of the customer quickly and easily. All the instruments are connected online to the bioprocess and deliver a continuous measurement signal.
\end{abstract}

Keywords: Biosensors · Biotechnology · Cell culture - Chemical sensors · Fermentation · Process control · Sensorix AG

\section{Introduction}

Monitoring fermentation and cell culture processes plays an important role in the biotech industry. Development and optimization of bioprocesses are very costly, both in time and money. Furthermore, process efficiency is directly dependant on precise bioprocess control and also quality assurance needs an accurate process monitoring to accomplish the demanding production protocols.

Today, sensors for $\mathrm{pH}$ and $\mathrm{O}_{2}$ are well established and are mostly used in situ, due to their robustness against common sterili-

${ }^{*}$ Correspondence: Dr. M. Rothmaier

SENSORIX AG

Technoparkstrasse 1

CH-8005 Zürich

Tel.: +4114451246

Fax: +4114451247

E-Mail: info@sensorix.com

www.sensorix.ch zation procedures. However, continuous control of feed parameters (e.g. glucose or methanol) and metabolites (e.g. lactate or acetate) is mostly lacking. Traditionally, samples are taken manually from time to time and analyzed in commercially available offline analyzers or with laboratory instruments (e.g. gas or liquid chromatography or disposable photometric test kits), respectively. However, offline analytics does not deliver the speed for measurement and control needed in bacteria or yeast fermentation and does not allow a precise control for long-term processes. Furthermore, manual sample taking, preparation and transportation is often time consuming and error prone. Therefore, only online and continuous process control will guarantee a safe, cost efficient and productive process management.

\section{Company Profile}

SENSORIX provides analytical solutions for process development, optimization, and control in biotechnology. SENSORIX was founded in 1999 as a spinoff company of the Swiss Federal Institute of Technology in Zürich (ETHZ) by five busi- ness partners. For two and a half years SENSORIX was a subtenant for office and laboratory space of ETHZ until it moved to its new commercial premises inside the Technopark Zurich. At the present time, SENSORIX employs 20 persons in Switzerland, the United Kingdom, Germany, and the USA and founded a subsidiary company, SENSORIX Inc., New Jersey (USA), last year.

\section{Product and Markets}

Only a few instruments for online and continuous process control are available on the market, but nearly all of them are limited to monitoring a single analyte only. In most cases chemical sensors or biosensors are used, due to their ability to measure reversibly concentration changes in liquids and gases. Furthermore, they offer the possibility for low maintenance cost, ease of use and are easy to automate.

SENSORIX first products, called SENSORIX Singlechannel 100 (SC 100) and Multichannel 100/200/300 (MC 100/200/ 300 ), respectively, are fully automated and autonomously working analytical instruments. Sample taking from the customer's 
process, analysis, and data output all work without an operator's intervention over several days up to four weeks. The MC and SC product line consist of a platform design for simultaneous operation of biosensors and chemical sensors. Different sensor technologies (amperometric, potentiometric, and optical signal transduction) can be assembled to analyze the same specimen. All sensors consist of a flow-through module, which incorporates a thin channel for liquid transport, the sensor element, and a connector to optical or electrical amplifier electronics. Thanks to the identical housings, all sensors can be combined with each other in series or in parallel giving the user the highest possible flexibility and allowing the operation of completely new combinations of sensors with different transduction principles within one analytical device at the same time. This multi-parameter or multidimensional analysis allows the creation of a fingerprint profile for the bioprocess (e.g. for validation use). SENSORIX has developed so far sensors for glucose, lactate, glutamate, glutamine, methanol, ethanol, ammonium, and acetate for continuous operation inside its instruments.

The SENSORIX SC or MC instruments are connected directly to the customer's process through a sterile sampling system, which reduces the risk of contamination for the fermentor and staff and maintains the sterility of the process (no mechanical parts like valves are opened and closed). The sampling system also holds back the cells or microorganisms and delivers a clean specimen of the cultivation media. Liquid that has been analyzed will be directed into the waste - consequently, a backflow to the customer's process is prevented. Up to nine feed parameters or metabolites can be monitored simultaneously and a sophisticated data analysis is delivered to the operator or to the customer's own process control software, respectively (Fig.). Thanks to continuous data acquisition the most advantageous operation mode, where sensor signals can be used to trigger an action, is also possible for fast processes. For these purposes analog and digital interfaces are available at the instrument's periphery and allow the users to develop their own feedforward bioprocess control.

\section{Future Trends}

Online analytics for process control in biotechnology will face several new challenges in the near future. Besides measuring feed parameters and metabolites the quantification of the preferred product (e.g. a protein) is still lacking and will not be accessible through classical sensor technology. New sensor transduction principles (e.g. mass measurements with coated cantilever arrays or biochips based on surface acoustic wave principles) will have to be developed to match the high demands in an industrial environment. Moreover, the trend to establish bioprocesses in small volumes and tiny vessels (e.g. titer plates) will not allow the connection of analytical instruments direct- ly through conventional sampling systems. New miniaturized probes will have to be developed to obtain the analytical information without interfering with the production environment or changing the production media (e.g. spectroscopic techniques like FT-IR).

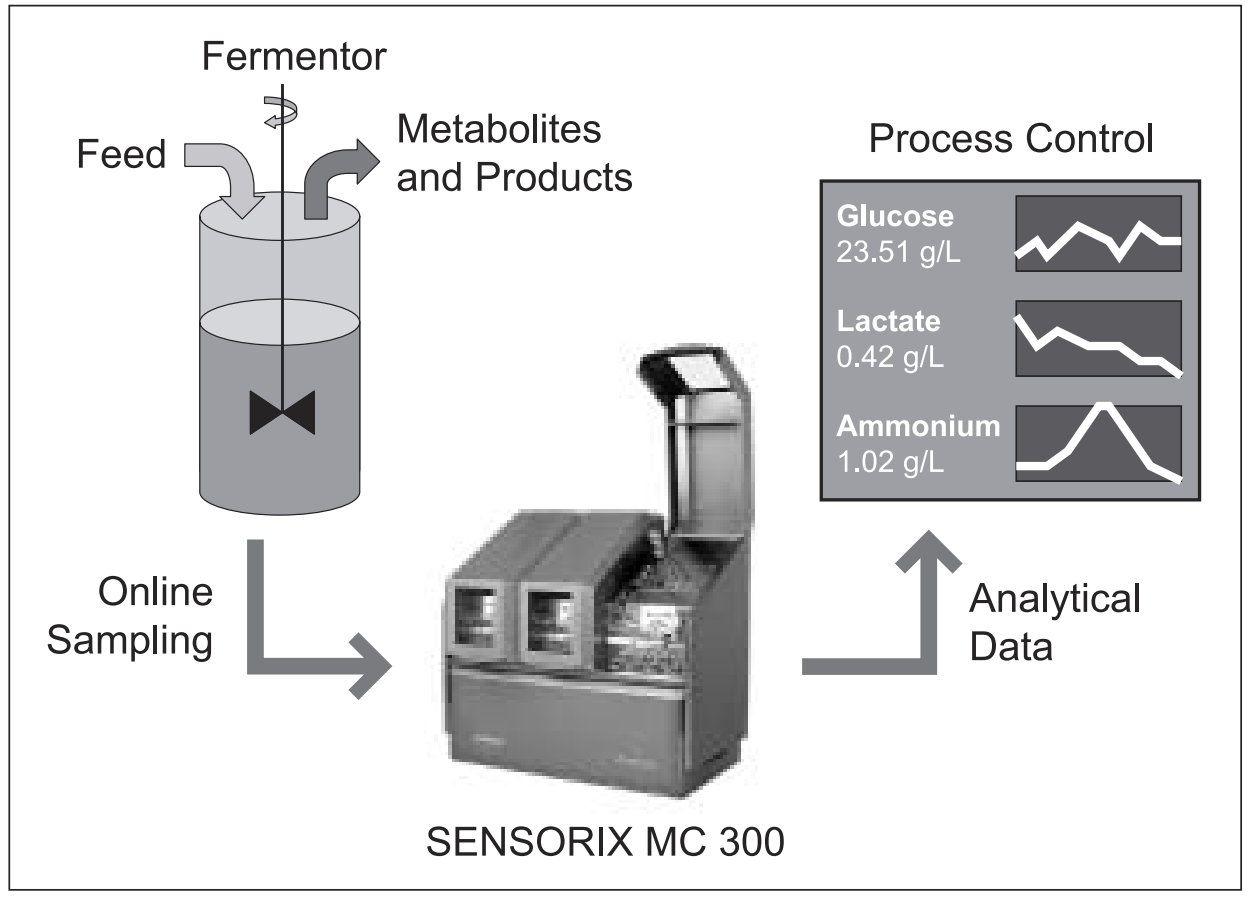

Fig. SENSORIX Multichannel 300 connected via a sterile sampling system to a fermentor. Analytical data will be collected, displayed and stored locally or delivered to the customer's process control software. 